University of Wollongong

Research Online

Faculty of Engineering and Information

Faculty of Engineering and Information

Sciences - Papers: Part A

Sciences

$1-1-2016$

\title{
Analysis of the magnetic torque on a tilted permanent magnet for drug delivery in capsule robots
}

Fredy Munoz

University of Wollongong, ffm517@uowmail.edu.au

Gursel Alici

University of Wollongong, gursel@uow.edu.au

Hao Zhou

University of Wollongong, hzhou@uow.edu.au

Weihua Li

University of Wollongong, weihuali@uow.edu.au

Metin Sitti

MaxPlanck Institute, sitti@is.mpg.de

Follow this and additional works at: https://ro.uow.edu.au/eispapers

Part of the Engineering Commons, and the Science and Technology Studies Commons

Research Online is the open access institutional repository for the University of Wollongong. For further information contact the UOW Library: research-pubs@uow.edu.au 


\title{
Analysis of the magnetic torque on a tilted permanent magnet for drug delivery in capsule robots
}

\author{
Abstract \\ In this paper, we present the analysis of the torque transmitted to a tilted permanent magnet that is to be \\ embedded in a capsule robot to achieve targeted drug delivery. This analysis is carried out by using an \\ analytical model and experimental results for a small cubic permanent magnet that is driven by an \\ external magnetic system made of an array of arc-shaped permanent magnets (ASMs). Our experimental \\ results, which are in agreement with the analytical results, show that the cubic permanent magnet can \\ safely be actuated for inclinations lower than $75^{\circ}$ without having to make positional adjustments in the \\ external magnetic system. We have found that with further inclinations, the cubic permanent magnet to \\ be embedded in a drug delivery mechanism may stall. When it stalls, the external magnetic system's \\ position and orientation would have to be adjusted to actuate the cubic permanent magnet and the drug \\ release mechanism. This analysis of the transmitted torque is helpful for the development of real-time \\ control strategies for magnetically articulated devices.

\section{Disciplines} \\ Engineering | Science and Technology Studies

\section{Publication Details} \\ Munoz, F., Alici, G., Zhou, H., Li, W. \& Sitti, M. (2016). Analysis of the magnetic torque on a tilted permanent \\ magnet for drug delivery in capsule robots. 2016 IEEE International Conference on Advanced Intelligent \\ Mechatronics (AIM) (pp. 1386-1391). USA: IEEE.
}




\title{
Analysis of the Magnetic Torque on a Tilted Permanent Magnet for Drug Delivery in Capsule Robots
}

\author{
Fredy Munoz, Gursel Alici, Hao Zhou, Weihua Li, and Metin Sitti
}

\begin{abstract}
In this paper, we present the analysis of the torque transmitted to a tilted permanent magnet that is to be embedded in a capsule robot to achieve targeted drug delivery. This analysis is carried out by using an analytical model and experimental results for a small cubic permanent magnet that is driven by an external magnetic system made of an array of arc-shaped permanent magnets (ASMs). Our experimental results, which are in agreement with the analytical results, show that the cubic permanent magnet can safely be actuated for inclinations lower than $75^{\circ}$ without having to make positional adjustments in the external magnetic system. We have found that with further inclinations, the cubic permanent magnet to be embedded in a drug delivery mechanism may stall. When it stalls, the external magnetic system's position and orientation would have to be adjusted to actuate the cubic permanent magnet and the drug release mechanism. This analysis of the transmitted torque is helpful for the development of real-time control strategies for magnetically articulated devices.
\end{abstract}

\section{INTRODUCTION}

Magnetic devices that can remotely be guided and actuated inside biological tissues have become highly attractive due to their potential benefits for minimally invasive procedures [1, 2]. These magnetic devices usually have internal permanent magnets (IPMs) that interact with an external magnetic system that generates a magnetic field that is harmless to living tissues [3]. Such interactions mainly consist of magnetic forces and torques imparted to IPMs for the actuation of needles [4], and other on-board modules on capsule endoscopes such as drug delivery [5, 6], biopsy [3, 7] and wireless insufflation [8]. Although magnetic actuation with permanent magnets overcomes the problem of power required by on-board mechanisms on magnetic devices [9], which has been demonstrated in a number of prototypes for capsule endoscopy [10-12], the complexity of the magnetic field and its interactions with magnetic devices still present challenges for real-time control [13].

Fredy Munoz and Hao Zhou are with the Intelligent Nano-Tera Systems Research Laboratory, University of Wollongong, Australia. E-mail: ffm517@uowmail.edu.au,hzhou@uow.edu.au

Gursel Alici (corresponding author) is with the School of Mechanical, Material and Mechatronic Engineering, University of Wollongong, Wollongong, NSW 2522, Australia. Tel.: +61 2-4221-4145; fax: +61 24221-5474, E-mail address: gursel@uow.edu.au.

Weihua Li is with the School of Mechanical, Material and Mechatronic Engineering, University of Wollongong, Wollongong, NSW 2522, Australia. E-mail: weihuali@uow.edu.au.

Metin Sitti is with Max Planck Institute for Intelligent Systems, Stuttgart, Germany, sitti@is.mpg.de.
This complexity has been formally analyzed for the interaction of a single external permanent magnet (EPM) that remotely actuates magnetic devices located at large distances [13-15]. These studies demonstrate that it is possible to develop real-time control strategies to remotely manipulate magnetic devices that have arbitrary position and/or orientation relative to the single EPM. However, the complexity of the magnetic interactions may increase, for the purpose of achieving real-time control, if the external magnetic system is made of multiple EPMs rather than a single permanent magnet.

In this paper, we aim to analyze the magnetic torque transmitted to an IPM that is arbitrarily orientated and actuated by an array of EPMs. Understanding the effects of the IPM's orientation on its transmitted torque is important for the development of real-time control strategies for magnetic devices. We specifically conduct this analysis for an IPM that is to be embedded in a slider-crank mechanism for targeted drug delivery in capsule robots. The capsule robot will travel through the digestive system, and its IPM's orientation will continuously change, affecting the torque transmitted to it by the EPMs. Therefore, the analysis of the transmitted torque for an arbitrary orientation of the IPM is important because it will allow the clinician to make any adjustments needed in the external magnetic system for an effective real-time control of the drug delivery system to be embedded in the capsule robot. To the best of our knowledge, this is the first time that such analysis is carried out for a drug delivery system in capsule robots.

The remainder of this paper is organized as follows. Section II provides the details of the overall system under study. Section III presents the theoretical methods for the analysis of the torque transmitted to a tilted IPM. Section IV provides the verification of the theoretical results with experimental results for flux density, magnetic torque and rotational movement of the IPM and the crank to release drug. Finally, discussions of the results and future work are presented in Section V.

Figure 1 shows a ring-shaped external magnetic system made of EPMs, the position (XYZ) and orientation $\left(\theta_{\mathrm{EPM}}, \varphi\right)$ of which can be controlled from a joystick. A rotating magnetic field around the patient bed is generated when the EPMs are physically rotated about the $\mathrm{z}$ axis. In this work, we have used an external magnetic system made of an array of 24 arc-shaped permanent magnets (ASMs) that we have optimized to generate a theoretical value of 500 $\mathrm{mT}$ at the centre of the system $(\mathrm{x}=\mathrm{y}=\mathrm{z}=0)$ as shown in Fig. 2. Each ASM has a radial thickness of $20 \mathrm{~mm}$ (with the inner radius $\mathrm{r}_{1}$ of $30 \mathrm{~mm}$ ), an axial length of $30 \mathrm{~mm}$ and an angular 
span of $30^{\circ}$. Therefore, an array of 12 ASMs is placed on top of another array of 12 ASMs, making a total length of $\Delta \mathrm{z}=60$ $\mathrm{mm}$ and the plane $\mathrm{z}=0$ is located in between the two arrays of ASMs. The magnetization grade of each ASM is 1.32 [T] (i.e., N45). Cubic and cylindrical IPMs are commonly used for magnetic actuation in medical applications [6, 10-12, 16]. In this work, a cubic IPM $(3.1 \mathrm{~mm})$ with a magnetization grade of 1.4 [T] (i.e., N50) is considered to be placed in a prototype of a capsule robot.

\section{OVERALL SYSTEM}

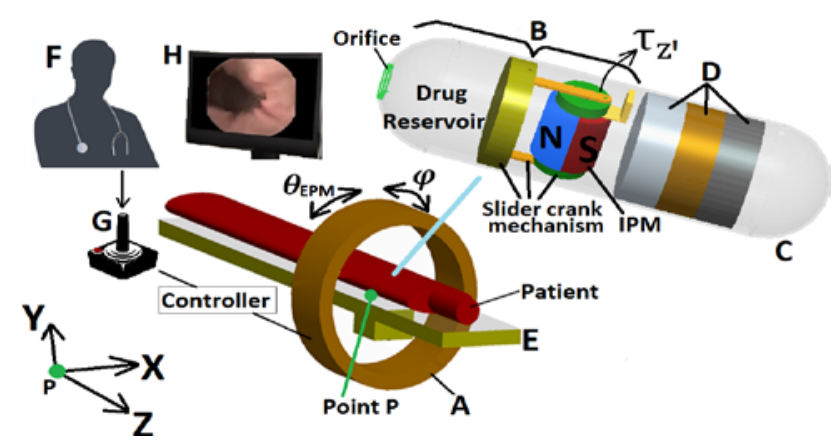

Figure 1. The main components of the proposed drug delivery system for WCE. A: ring-shaped external magnetic system, B: drug release module, C: the capsule robot, D: complementary modules within the capsule (anchoring mechanism, active locomotion system and localization and orientation detection module), E: patient bed, F: clinician, G: joystick, H: Human Capsule Interface. Point $P$ represents the origin of the general coordinate system XYZ, $\theta_{\mathrm{EPM}}$ is taken with respect to the $\mathrm{x}$ axis, and $\varphi$ is taken with respect to the $\mathrm{z}$ axis.

The capsule robot is to operate within the cylindrical region of interest defined by the maximum radial operating distance $r_{1}$ and the length of $\Delta \mathrm{z}=60 \mathrm{~mm}$. Since the drug release mechanism is actuated by a magnetic torque, the operating distance $r_{1}$ can be increased to meet the requirements of a more realistic medical application (i.e., $\mathrm{r}_{1}>120 \mathrm{~mm}$ ) while the dimensions of the external magnetic system are scaled up at the same time [13, 17].

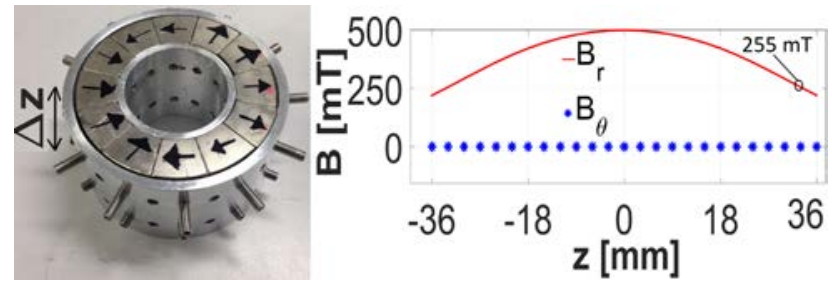

Figure 2. External magnetic system made of 24 ASMs and the magnitude of the three components of the flux density $\mathbf{B}$ along the $\mathrm{z}$ axis. $|\mathbf{B}|=\mathrm{B}_{\mathrm{r}}=\mathrm{B}_{\mathrm{x}}$ and $\mathrm{B}_{\theta}=\mathrm{B}_{\mathrm{z}}=0$ along the $\mathrm{z}$ axis, for $\theta_{\mathrm{EPM}}=0 .|\mathbf{B}|=255[\mathrm{mT}]$ at $\mathrm{x}=\mathrm{y}=0, \mathrm{z}=33 \mathrm{~mm}$.

A magnetic torque $\tau_{\mathrm{z} \text {, }}$, as shown in Fig. 1, will be imparted to the IPM embedded in the capsule robot as its magnetization vector $\mathbf{m}$ interacts with the rotating magnetic field created by the ASMs. The transmitted torque $\tau_{z}$ is then converted into a piston force $\mathbf{F}$ by means of a slider-crank mechanism that is physically connected to the IPM. In this way, a piston will push drug out of a reservoir when the ASMs are rotated around the patient's body as shown in Fig.
1. Within the capsule robot, we define a coordinate system $X^{\prime} Y^{\prime} Z^{\prime}$, the origin of which coincides with the IPM's centre. When the IPM's centre coincides with the centre of ASMs and the axes of the two coordinate systems are aligned, it is possible to approximately transmit a magnetic peak torque $\tau_{\mathrm{z}}$ of $3.5[\mathrm{mNm}]$ on a $3.1 \mathrm{~mm}$ cubic IPM by using only 4 ASMs that generate approximately $114[\mathrm{mT}]$ at the IPM's centre [17]. However, in a real application, the IPM can be off the centre and/or tilted as it will move along with the capsule robot. Understanding the effects of the changes in the IPM's position and orientation within the cylindrical region of interest on $\tau_{\mathrm{z}}$ is important for the development of real-time control strategies for magnetic devices. In this work, we present how $\tau_{\mathrm{z}}$, is affected by changes in the IPM's orientation with the IPM's centre restricted to points along the $\mathrm{z}$ axis. In Section III, we present the methodology used to conduct such assessment by means of analytical functions that model the magnetic flux density $\mathbf{B}$ created by the ASMs and the magnetic torque $\tau_{z^{\prime}}$.

\section{THEORETICAL METHODS}

The magnetic torque $\boldsymbol{\tau}$, transmitted to the IPM with a volume $\mathrm{V}$ and a magnetization vector $\mathbf{m}$ that is exposed to an adjustable external magnetic flux density $\mathbf{B}$ is given by [18]

$$
\boldsymbol{\tau}=\mathrm{V}(\mathbf{m} \times \mathbf{B}) / \mu_{0} \quad[\mathrm{Nm}]
$$

$\mu_{0}$ is the permeability of the free space (i.e., $4 \pi 10^{-7} \mathrm{H} /$ $\mathrm{m})$; and the units for both magnetization and magnetic flux density are Tesla. $\mathbf{B}$ is the magnetic flux density generated by the external magnetic system and computed at the centre of the IPM. The torque $\boldsymbol{\tau}$ will tend to orient the vector $\mathbf{m}$ along $\mathbf{B}$ and can generate a rotational movement on the IPM. For an external magnetic system made of an array of ASMs, B, which can be accurately computed at any point with the Amperian current model, is expressed in cylindrical coordinates as [19]

$$
\mathbf{B}=\mathrm{B}_{\mathrm{r}} \mathbf{u}_{\mathbf{r}}+\mathrm{B}_{\theta} \mathbf{u}_{\boldsymbol{\theta}}+\mathrm{B}_{\mathrm{z}} \mathbf{u}_{\mathbf{z}}
$$

B represents a static magnetic flux density that becomes a rotating magnetic flux density when the ASMs are rotated about the $\mathrm{z}$ axis. We are interested in estimating $\tau_{\mathrm{z}}$ acting on the IPM that is tilted by an angle $\theta_{z}$ as shown in Fig. 3 a). For this purpose, we choose a coordinate system $\mathrm{X}^{\prime} \mathrm{Y}^{\prime} \mathrm{Z}^{\prime}$ as shown in Fig. 3 a). Therefore, the IPM's magnetization vector $\mathbf{m}$ can rotate about the $\mathrm{z}^{\prime}$ axis in a circular trajectory with an orientation $\theta_{\text {IPM }}$. We can express the projections of $\mathbf{m}$ in the plane $X^{\prime} Y^{\prime}$ as

$$
\mathbf{m}=|\mathbf{m}|\left(\cos \theta_{\mathrm{IPM}} \mathbf{u}_{\mathbf{x}^{\prime}}+\sin \theta_{\mathrm{IPM}} \mathbf{u}_{\mathbf{y}^{\prime}}\right)
$$

Our external magnetic system generates a rotating $\mathbf{B}$ at any point along the $\mathrm{z}$ axis as shown in Fig. $3 \mathrm{~b}$ ). Since $\mathrm{B}_{\mathrm{z}}=0$ along the $\mathrm{z}$ axis, when the external magnetic system is rotated by an angle $\theta_{\mathrm{EPM}}$, the projection of $\mathbf{B}$ in any plane that is parallel to the plane $\mathrm{XY}$ can be expressed as

$$
\mathbf{B}=|\mathbf{B}|\left(\cos \theta_{\mathrm{EPM}} \mathbf{u}_{\mathbf{x}}+\sin \theta_{\mathrm{EPM}} \mathbf{u}_{\mathbf{y}}\right)
$$


However, we can also find the 3 projections of $\mathbf{B}$ in the system $\mathrm{X}^{\prime} \mathrm{Y}^{\prime} \mathrm{Z}^{\prime}$ as

$$
\mathbf{B}=\mathrm{B}_{\mathrm{x}^{\prime}} \mathbf{u}_{\mathbf{x}^{\prime}}+\mathrm{B}_{\mathrm{y},} \mathbf{u}_{\mathbf{y}^{\prime}}+\mathrm{B}_{\mathrm{z}^{\prime}} \mathbf{u}_{\mathrm{z}^{\prime}}
$$

where

$$
\begin{aligned}
\mathrm{B}_{\mathrm{x}^{\prime}} & =|\mathbf{B}| \cos \theta_{\mathrm{EPM}} \cos \theta_{\mathrm{z}} \\
\mathrm{B}_{\mathrm{y}^{\prime}} & =|\mathbf{B}| \sin \theta_{\mathrm{EPM}} \\
\mathrm{B}_{\mathrm{z}^{\prime}} & =|\mathbf{B}| \cos \theta_{\mathrm{EPM}} \sin \theta_{\mathrm{z}}
\end{aligned}
$$

The magnetic torque $\tau_{\mathrm{z}^{\prime}}$, which is the axial component of $\boldsymbol{\tau}^{\prime}$ (i.e., the magnetic torque with respect to the system $\mathrm{X}^{\prime} \mathrm{Y}^{\prime} \mathrm{Z}^{\prime}$ ), is only affected by $\mathbf{B}_{\mathbf{x}^{\prime} \mathbf{y}}$, which represents the projections of $\mathbf{B}$ in the plane $X^{\prime} Y^{\prime}$ and is expressed as

$$
\mathbf{B}_{\mathrm{x}^{\prime} \mathbf{y}^{\prime}}=\mathrm{B}_{\mathrm{x}^{\prime}} \mathbf{u}_{\mathrm{x}^{\prime}}+\mathrm{B}_{\mathrm{y}^{\prime}} \mathbf{u}_{\mathbf{y}^{\prime}}
$$

By substituting Eq. (3) and Eq. (9) into Eq. (1) and only taking the axial component of $\boldsymbol{\tau}^{\prime}$, we obtain

$$
\tau_{\mathrm{z}^{\prime}}=\frac{\mathrm{v}}{\mu_{0}}|\mathbf{m}|\left(\mathrm{B}_{\mathrm{y}^{\prime}} \cos \theta_{\mathrm{IPM}}-\mathrm{B}_{\mathrm{x}} \sin \theta_{\mathrm{IPM}}\right)
$$

By substituting Eq. (6) and Eq. (7) into Eq. (10), we find the magnetic torque about the IPM's axial axis as

$$
\begin{aligned}
\tau_{\mathrm{z} \prime}= & \frac{\mathrm{v}}{\mu_{0}}|\mathbf{m}||\mathbf{B}|\left(\sin \theta_{\mathrm{EPM}} \cos \theta_{\mathrm{IPM}}-\right. \\
& \left.\cos \theta_{\mathrm{EPM}} \cos \theta_{\mathrm{z}} \sin \theta_{\mathrm{IPM}}\right)
\end{aligned}
$$

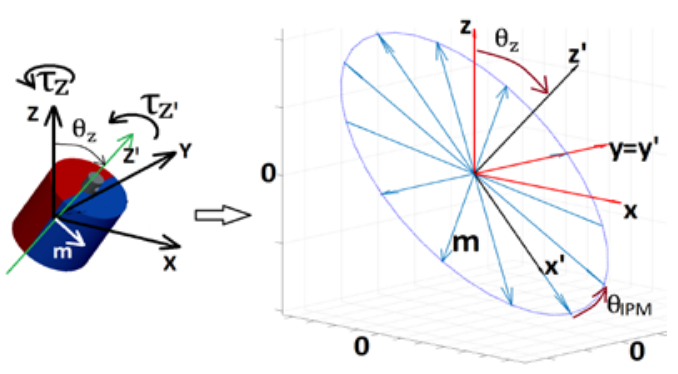

a)

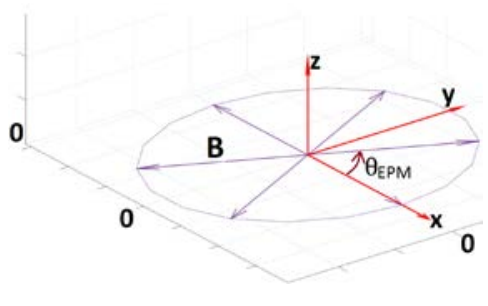

b)

Figure 3. a) IPM inclined by an angle $\theta_{\mathrm{z}}$ and $\mathbf{m}$ rotates about the $\mathrm{z}^{\prime}$ axis in a circular trajectory, b) the rotating magnetic flux density $\mathbf{B}$ at any point along the $\mathrm{z}$ axis (i.e., $\mathrm{B}_{\mathrm{z}}=0$ ).

Eq. (11) indicates that the torque $\tau_{\mathrm{z}}$, depends on the orientation of the IPM (i.e., $\theta_{\text {IPM }}$ and $\theta_{\mathrm{z}}$ ) and the rotation of the ASMs (i.e., $\left.\theta_{\mathrm{EPM}}\right)$. V, $|\mathbf{m}|$ and $|\mathbf{B}|$ are known and the latter varies along the $\mathrm{z}$ axis, for $\theta_{\mathrm{EPM}}=0$, as shown in Fig. 2 .
In order to analyze the effects of $\theta_{\text {IPM }}, \theta_{\mathrm{EPM}}$ and $\theta_{\mathrm{z}}$ on $\tau_{\mathrm{z}}$ at any point on the $\mathrm{z}$ axis, we firstly make the IPM's centre to coincide with the point $x=0, y=0, z=33 \mathrm{~mm}$. At this specific point, the magnitude of the magnetic flux density is 255 [mT]. Since Eqs. (4) and (11) are valid at any point along the $\mathrm{z}$ axis, an arbitrary position for the IPM's centre along the $\mathrm{z}$ axis can be chosen to conduct this analysis. However, the selection of this specific point facilitates the collection of experimental data, as presented in Section IV. Secondly, we let $\theta_{\mathrm{z}}$ to take the values of $0^{\circ}, 30^{\circ}, 60^{\circ}, 75^{\circ}$ and $90^{\circ}$, and finally we observe $\tau_{\mathrm{z}}$ as a function of $\theta_{\mathrm{IPM}}$ and $\theta_{\mathrm{EPM}}$ for each value of $\theta_{\mathrm{z}}$. Figs. 4-5 show these results for $\theta_{\mathrm{z}}=0^{0}$ and $90^{\circ}$, respectively. The results highlighted in the red and black curves represent $\tau_{\mathrm{z}}$, for $\theta_{\mathrm{IPM}}=0^{\circ}$ and $90^{\circ}$, respectively.

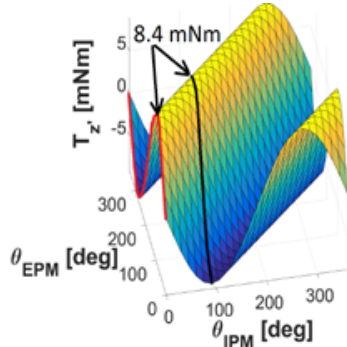

a)

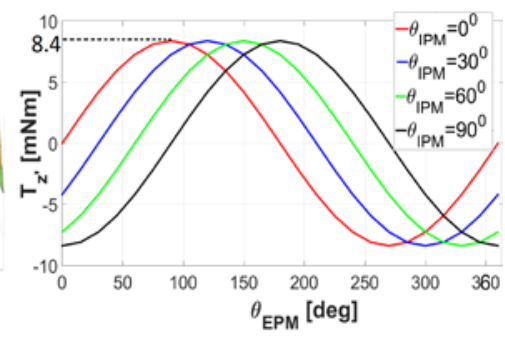

b)
Figure 4. Magnetic torque $\tau_{\mathrm{z}}$, for: a) $\theta_{\mathrm{z}}=0^{\circ}$, b) $\theta_{\mathrm{z}}=0^{\circ}$ and $0^{0} \leq \theta_{\text {IPM }} \leq 90^{\circ}$.

When the IPM is not tilted (i.e., $\theta_{\mathrm{z}}=0^{0}$ ), a peak torque of $\tau_{\mathrm{z}}=8.4[\mathrm{mNm}]$ is always obtained regardless of the orientation $\theta_{\text {IPM }}$ of the magnetization vector $\mathbf{m}$ as shown in Fig. 4. However, this peak torque decreases as $\theta_{z}$ increases and also with the increments of $\theta_{\text {IPM }}$. According to the results shown in Fig. 5, $\theta_{\mathrm{IPM}}=90^{\circ}$ and $270^{\circ}$ (i.e., when $\mathbf{m}$ is aligned with the y axis) are critical angles where a minimum peak torque of $0[\mathrm{mNm}]$ is obtained. At these angles, the IPM and therefore, the slider-crank mechanism will stall. However, if $\theta_{\text {IPM }}$ is less than $75^{\circ}$, a minimum peak torque of $2.2[\mathrm{mNm}]$ can be guaranteed on the IPM even at an IPM's inclination of $\theta_{\mathrm{z}}=90^{\circ}$. This torque can still be sufficient to actuate the drug release mechanism.

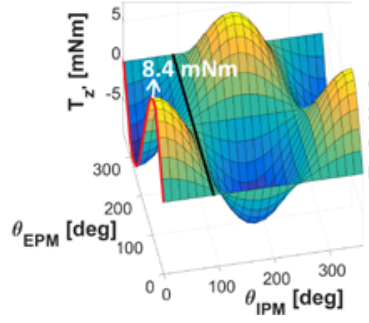

a)

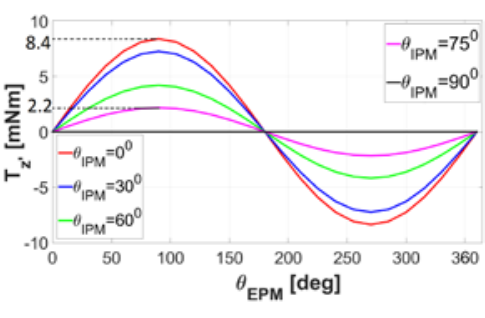

b)
Figure 5. $\tau_{\mathrm{z}}$, for a) $\theta_{\mathrm{z}}=90^{\circ}$, b) $\theta_{\mathrm{z}}=90^{\circ}$ and $0^{\circ} \leq \theta_{\mathrm{IPM}} \leq 90^{\circ}$ (peak torque of $4.2[\mathrm{mNm}]$ at $\theta_{\mathrm{IPM}}=60^{\circ}$ ).

A peak torque of approximately $3.5[\mathrm{mNm}]$ has been previously estimated as an appropriate magnitude to release drugs in capsule robots $[17,19]$. Therefore, we conclude that even if the IPM is tilted by $\theta_{z}=90^{\circ}$, the external magnetic 
system can be rotated in a way that $\theta_{\text {IPM }}$ reaches a maximum angle of $60^{\circ}$ or $75^{\circ}$ and the transmitted torque will be approximately 4.2 or 2.2 [mNm] (as shown in Fig. 5), respectively. However, if higher peak torques were required, the IPM's centre can be moved towards the centre of the system where higher magnetic flux densities are expected. For example, a peak torque of $8.4 \mathrm{mNm}$ is obtained when the IPM's centre is placed at $\mathrm{z}=33 \mathrm{~mm} \quad(\mathrm{x}=\mathrm{y}=0)$. A maximum peak torque can be obtained when $|\mathbf{B}|=500[\mathrm{mT}]$ right at the centre of the system as shown in Fig. 2. We also find results similar to those shown in Figs. 4-5 for $90^{\circ}<\theta_{\mathrm{z}}<360^{\circ}$ due to the symmetry of the magnetic flux density. Therefore, the analysis of the magnitude of $\tau_{z \prime}$ for $0^{0} \leq \theta_{\mathrm{z}} \leq 90^{0}$ is sufficient. These theoretical results are validated with the experimental results in Section IV.

\section{EXPERIMENTAL METHODS}

A 3-channel Gauss meter (Lakeshore-Model 460) was used to measure the magnetic flux density $\mathbf{B}$. A torque gauge (HTG2-40 supplied by IMADA) with its respective torque sensor held the IPM in place to measure the transmitted torque $\tau_{z}$. The IPM was connected to the torque sensor via a plastic connector that was prototyped using a 3D printer. Additionally, we fabricated plastic angular guides that allowed us to incline the IPM with the angles of $\theta_{z}=30^{\circ}, 60^{\circ}$, $75^{\circ}$ and $90^{\circ}$. The angular guides and the probe tip of the Gauss meter can be moved along the $\mathrm{X}, \mathrm{Y}$ and $\mathrm{Z}$ axes. These displacements are controlled by a micromanipulation system constructed of XYZ stages as shown in Fig. 6.

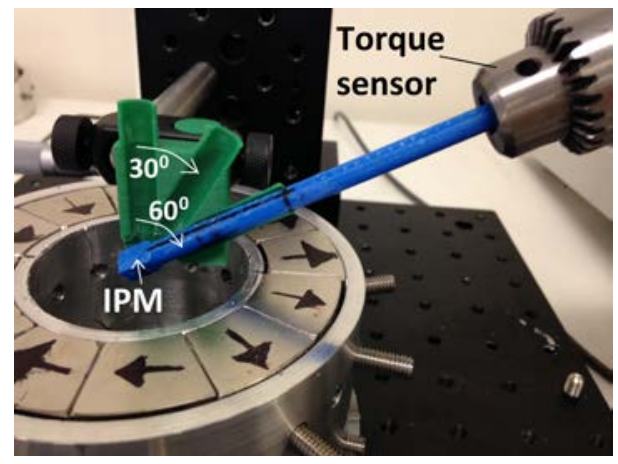

Figure 6. Experimental setup to measure $\tau_{\mathrm{z}}$ imparted by the array of ASMs: angular guide, mounted on the micromanipulation system, to tilt the IPM by $\theta_{z}=30^{\circ}$ or $60^{\circ}$ (a similar guide was fabricated to allow inclinations of $75^{\circ}$ and $90^{\circ}$ ). The IPM's centre is placed at $\mathrm{x}=\mathrm{y}=0, \mathrm{z}=33 \mathrm{~mm}$ and tilted by $\theta_{\mathrm{z}}=60^{\circ}$.

\section{A. Magnetic Flux Density}

In the first set of experiments, we measured $\mathbf{B}$ along the $\mathbf{z}$ axis by fixing the external magnetic system (i.e., $\theta_{\mathrm{EPM}}=0$ at all times) and only moving the tip of the probe with increments of $3 \mathrm{~mm}$ from $-36 \mathrm{~mm}$ to $36 \mathrm{~mm}$ along the $\mathrm{z}$ axis ( $\mathrm{x}=\mathrm{y}=0$ at all times). Under these conditions, the cylindrical components of $\mathbf{B}$ can be expressed in Cartesian components as $B_{x}=B_{r}, B_{y}=B_{\theta}$ and $B_{z}=B_{z}$. Therefore, we measured $B_{x}, B_{y}$ and $B_{z}$ along the $z$ axis, although only $B_{x}$ is shown in Fig. 7 because $B_{y}$ and $B_{z}$ varied between $-3[\mathrm{mT}]$ and $3[\mathrm{mT}]$.
These small values of $B_{y}$ and $B_{z}$ can be taken as $0[\mathrm{mT}]$ for practical purposes because they will hardly contribute to the magnetic torque transmitted to the IPM. Therefore, the static magnetic flux density along the $\mathrm{z}$ axis $\mathbf{B}$ is equal to $B_{x}$ for $\theta_{\mathrm{EPM}}=0^{0}$.

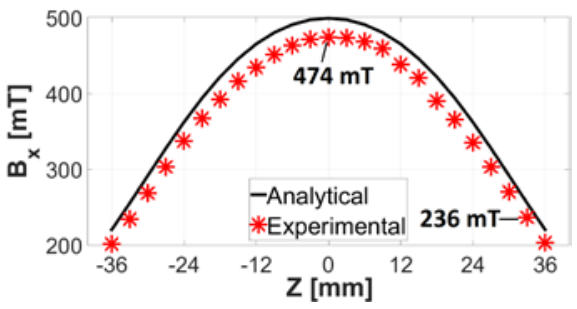

Figure 7. $B_{x}$ along the $z$ axis and $B_{y}=B_{z}= \pm 3[\mathrm{mT}]$ along the $z$ axis. $B_{x}=236$ $[\mathrm{mT}]$ at $\mathrm{x}=\mathrm{y}=0, \mathrm{z}=33 \mathrm{~mm}$.

When the external magnetic system was rotated (i.e., when $\theta_{\mathrm{EPM}}$ varied), $\mathbf{B}$ became a rotating magnetic flux density. These experimental results verified the accuracy of the analytical model for $\mathbf{B}$ expressed in Eq. (4) when the external magnetic system was rotated by an angle $\theta_{\text {EPM }}$. We believe that the difference between the analytical and experimental results shown in Fig. 7 (which were always less than $8.6 \%$ ) may be due to the small gaps between the ASMs and the aluminum case that holds them in place and also some small misalignments among the 24 ASMs. However, these differences can decrease the magnitude of $\mathbf{B}$ but do not change our model expressed in Eq. (4).

\section{B. Magnetic Torque}

In the second set of experiments, we firstly placed the IPM's centre at $\mathrm{x}=\mathrm{y}=0$ and $\mathrm{z}=33 \mathrm{~mm}$. This is a practical and convenient point that has allowed us to tilt the IPM up to a maximum angle of $\theta_{z}=90^{\circ}$. For a larger inclination (or if the IPM's centre was located at points such $\mathrm{z}<33 \mathrm{~mm}$ ), the plastic connector attached to the torque sensor contacted the external magnetic system and impeded the direct measurement of $\tau_{z^{\prime}}$. Similarly, points above $\mathrm{z}=33 \mathrm{~mm}$ were not of interest for two reasons: they are outside the cylindrical region of interest where the capsule robot would operate and the magnetic flux density was lower than 236 [mT], which would decrease the magnetic torque to smaller values that may not be measured by our torque sensor, especially when the IPM was tilted. Secondly, we were interested in measuring $\tau_{\mathrm{z}}$, when the IPM's magnetization vector was aligned with the $+y$ axis (i.e., $\theta_{\text {IPM }}=90^{\circ}$ ) since this is a critical angle for the transmitted torque $\tau_{z}$ as presented in Section III. Therefore, we conducted our second set of experiments to measure $\tau_{\mathrm{z}}$, as a function of $\theta_{\mathrm{EPM}}$ and $\theta_{\mathrm{z}}$ as shown in Fig. 8.

These experimental results validate the analytical model for $\tau_{z \prime}$ expressed in Eq. (11). According to these results, peak torques from 2 to $4[\mathrm{mNm}]$ were obtained when the IPM was tilted by an angle $\theta_{\mathrm{z}}$ between $75^{\circ}$ and $60^{\circ}$, respectively. Consequently, the IPM and the crank of the drug release module can be rotated even if the IPM is tilted by these angles. However, the peak torque of $\tau_{\mathrm{z}}$ continued 
decreasing if the IPM was further inclined (i.e., for $\theta_{\mathrm{z}}>75^{0}$ ), reaching $0[\mathrm{mNm}]$ at $\theta_{\mathrm{z}}=90^{\circ}$.

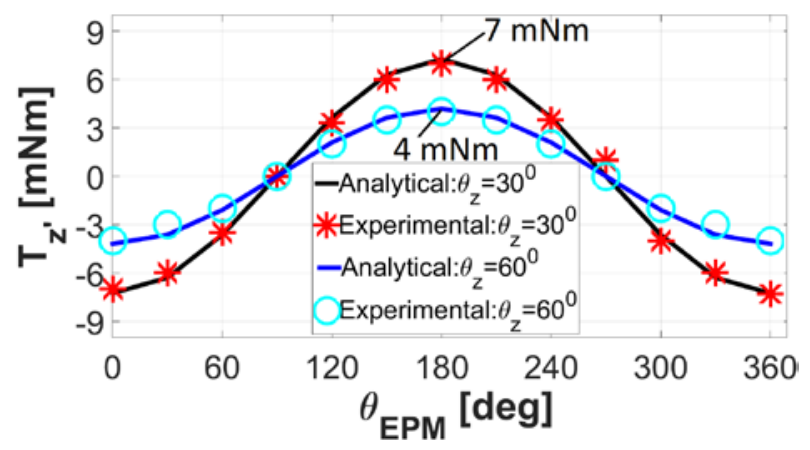

a)

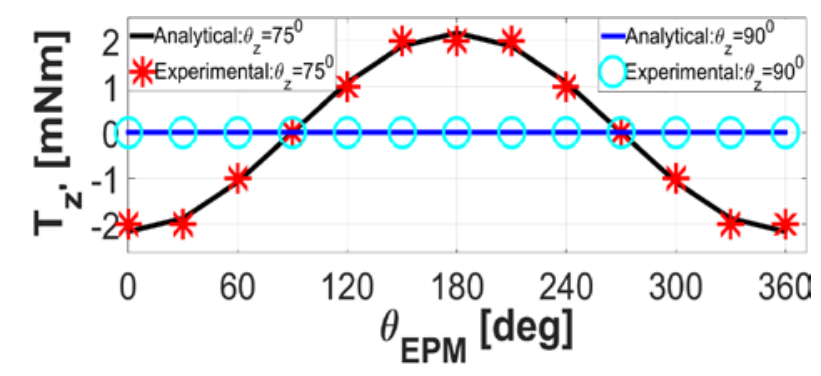

b)

Figure 8. Magnetic torque $\tau_{z}$, for inclinations: a) $\theta_{z}=30^{\circ}$ and $60^{\circ}$, b) $\theta_{z}=75^{0}$ and $90^{\circ}$. $\theta_{\text {IPM }}=90^{\circ}$ (i.e., $\mathbf{m}$ is aligned with the $+y$ axis).

Therefore, the IPM and the crank stall for any angle $\theta_{\mathrm{EPM}}$ if the IPM has the specific orientation determined by $\theta_{\mathrm{IPM}}=90^{\circ}$ (or $270^{\circ}$ ) and $\theta_{\mathrm{z}}=90^{\circ}$. At these values, the inclination $\varphi$ (see Fig. 1) and perhaps the position of the external magnetic system would need to be adjusted by the clinician to activate the drug release mechanism. Depending on the need to generate different drug profiles (i.e., changes in number of doses or changes in release rates), the clinicians may be able to follow different real-time control strategies for the capsule robot by moving the external magnetic system to tailor therapeutic treatments to individuals’ needs.

\section{The Slider-Crank Mechanism}

As shown in Fig. 9, we have inserted the cubic IPM into its IPM case (A) which is connected to the crank of the slider-crank mechanism. The IPM case is mounted on a platform (D) that has a small orifice through which the IPM case's axis was inserted (i.e., the crank shaft $\mathrm{C}$ ). In this way, the IPM and the crank can freely rotate about the $\mathrm{z}^{\prime}$ axis. Furthermore, the platform (D) is connected to a mobile frame (B) that can be inclined with respect to the $\mathrm{x}$ axis by fixing its orifice 1 and rotating its orifice 2 by an angle $\theta_{\mathrm{z}}$. All these components were fabricated using a $3 \mathrm{D}$ printer. We also fabricated the angular guides with orifices placed at $\theta_{z}=45^{\circ}, 75^{\circ}$ and $90^{\circ}$. These angular guides were mounted on a micromanipulation system as shown in Fig. 10.

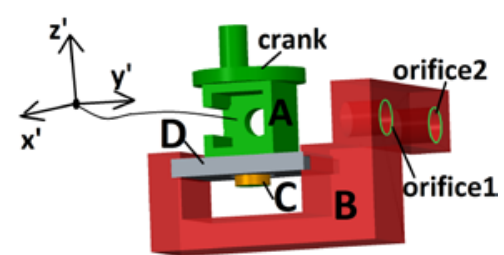

Figure 9. The disk-shaped crank is directly mounted on the IPM case (A). A: cubic IPM case, B: mobile frame with two orifices, C: the crank shaft that is aligned with the $\mathrm{z}^{\prime}$ axis, D: platform that supports the IPM case. The origin of the system $\mathrm{X}^{\prime} \mathrm{Y}^{\prime} \mathrm{Z}^{\prime}$ coincides with the centre of the IPM.

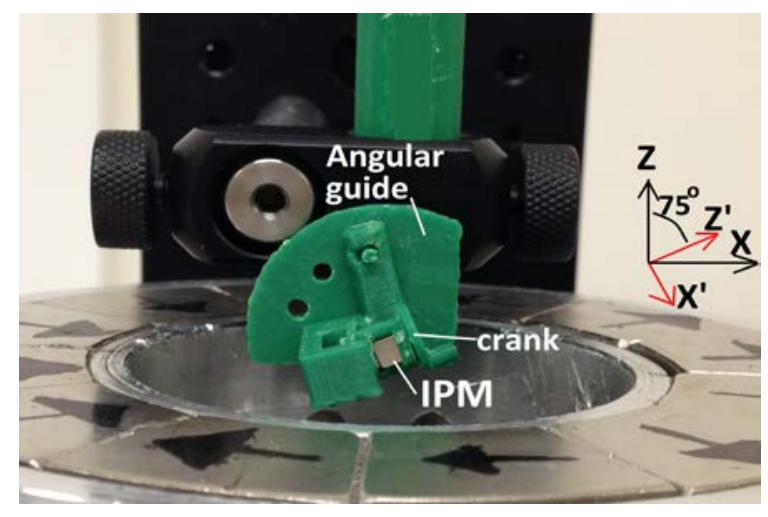

Figure10. The IPM and the crank are tilted by $\theta_{\mathrm{z}}=75^{\circ}$.

In the final set of experiments, we were interested in observing the critical angle of inclination by which the IPM and the crank would stall. We firstly placed the IPM's centre at $\mathrm{x}=\mathrm{y}=0$ and $\mathrm{z}=33 \mathrm{~mm}$, inclined the IPM by $\theta_{\mathrm{z}}=75^{\circ}$ and manually rotated the external magnetic system in the clockwise and counterclockwise directions. We observed that the IPM and the crank rotated in the same directions and never stalled regardless of the initial condition of $\theta_{\text {IPM }}$. Secondly, we moved the IPM's centre along the $\mathrm{z}$ axis towards the centre of the system (with the decrements of 10 $\mathrm{mm}$ ) and observed that the IPM and the crank freely rotated as the external magnetic system was rotated (again irrespective of the initial condition of $\theta_{\text {IPM }}$ ). The same process was conducted for $\theta_{\mathrm{z}}=45^{\circ}$ and the crank always rotated as it was driven by the rotation of the ASMs.

Thirdly, we placed the IPM's centre at $\mathrm{x}=\mathrm{y}=0$ and $\mathrm{z}=33$ $\mathrm{mm}$ but this time we inclined the IPM by $\theta_{\mathrm{z}}=90^{\circ}$ and made $\theta_{\mathrm{IPM}}=0^{0}$. Under these conditions, we started with the external magnetic system oriented at $\theta_{\mathrm{EPM}}=0^{0}$ and manually rotated it in the counterclockwise and clockwise directions until $\theta_{\mathrm{EPM}}$ reached approximately $30^{\circ}$ and $330^{\circ}$, respectively. We observed that the IPM and the crank rotated by approximately the same angles of $\theta_{\mathrm{IPM}}=30^{\circ}$ and $330^{\circ}$. However, when the external magnetic system was further rotated until it reached $\theta_{\mathrm{EPM}}=90^{\circ}$ (or $270^{\circ}$ ), we observed that the IPM and the crank also continued rotating until $\mathbf{m}$ was aligned with the y axis (i.e., $\theta_{\text {IPM }}$ reached $90^{\circ}$ or $270^{\circ}$ ) but the crank stalled at this angles as we continued rotating the external magnetic system.

Finally, we moved the IPM's centre along the $\mathrm{z}$ axis (with the decrements of $10 \mathrm{~mm}$ ) and at each point we maintained $\theta_{\mathrm{z}}=90^{\circ}$. We observed that the crank always stalled right at 
$\theta_{\mathrm{EPM}}=90^{\circ}$. These experimental results, which are in agreement with the theoretical results, indicate that for $\theta_{\mathrm{z}} \leq 75^{\circ}$, the crank was driven by only the rotation of the ASMs and no adjustments in the position and/or orientation of the external magnetic system were needed. However, as the IPM and the crank were further inclined, the transmitted torque decreased to values that could no longer actuate the crank. We found that the crank stalled when the IPM was tilted by $90^{\circ}$ and $\mathbf{m}$ was aligned with y axis $\left(\theta_{\mathrm{z}}=\right.$ $90^{\circ}, \theta_{\text {IPM }}=90^{\circ}$ or $270^{\circ}$ ). These angles can be used in a real-time control strategy when it may be desired not to actuate the drug release mechanism. For example, in a clinical application, the drug release module should not be actuated when the capsule robot is still travelling to the target location. Once the capsule robot reaches its target, the clinician can adjust the position and orientation of the ASMs to activate the drug release module and generate different drug profiles by controlling the release rate, release amount and number of doses in real time.

These experimental results are valid if the prototype of the external magnetic system is scaled up by a factor s and the operating distance is simultaneously increased by the same factor [17, 19]. Therefore, the analysis of the magnetic torque imparted to a tilted IPM that moves along the $\mathrm{z}$ axis presented in this study can be used in a clinical application to actuate a drug release mechanism embedded in a capsule robot. Furthermore, this study can be extended to remotely propelled devices requiring an external propulsion or articulation source.

\section{CONCLUSION AND FUtURE WORK}

In this paper, we have analyzed the magnetic torque transmitted to a $3.1 \mathrm{~mm}$ cubic IPM that is arbitrarily orientated and actuated by an array of ASMs. We find an analytical model for the torque transmitted to the IPM whose centre is located at any point along the $\mathrm{z}$ axis. We verified the accuracy of this analytical model with experimental results which showed that the IPM and therefore, the crank of the drug release mechanism could always be actuated if the IPM was tilted by angles lower than $75^{\circ}$. The crank stalled when the IPM was tilted by $90^{\circ}$ and its magnetization vector $\mathbf{m}$ was aligned with the y axis. Although the actuation of the IPM and the crank were guaranteed at the maximum angular inclination of $75^{\circ}$, we believe that when the piston of the drug delivery mechanism is articulated with the entire mechanical system and the drug is stored in its reservoir, additional friction forces and load will be present in the capsule robot. Therefore, we expect that the maximum angular inclination to guarantee the actuation of the drug release module should be below $75^{\circ}$. This will be investigated in our future work.

Another interesting result of this study is that although we aim to embed this IPM in a capsule robot to achieve drug delivery, the analysis of the transmitted torque on an IPM with an arbitrary orientation can be applied to any magnetic propulsion system requiring a remote transmission of a rotational magnetic field or torque to a target device. This analysis is important for the development of real-time control strategies for magnetic devices. In our future work, we will extend this analysis to any point within the cylindrical region of interest rather than only along the $\mathrm{z}$ axis.

\section{REFERENCES}

[1]C. D. Natali, J. Buzzi, N. Garbin, M. Beccani, and P. Valdastri, "ClosedLoop Control of Local Magnetic Actuation for Robotic Surgical Instruments," IEEE Transactions on Robotics, vol. 31, pp. 143-156, Feb. 2015.

[2]B. J. Nelson, L. K. Kaliakatsos, and J. J. Abbott, "Microrobots for Minimally Invasive Medicine," Annu. Rev. Biomed. Eng., vol. 12, pp. 5585, Apr. 2010.

[3] M. Simi, G. Gerboni, A. Menciassi, and P. Valdastri, "Magnetic torsion spring mechanism for a wireless biopsy capsule," ASME J. Med. Devices, vol. 7, Dec. 2013.

[4]P. Vartholomeos, C. Bergeles, L. Qin, and P. E. Dupont, "An MRIpowered and controlled actuator technology for tetherless robotic interventions "International Journal of Robotics Research vol. 32, pp. 1536-1552, Nov. 2013.

[5]F. Munoz, G. Alici, and W. Li, "A review of drug delivery systems for capsule endoscopy " Advanced Drug Delivery Reviews, vol. 71, pp. 77-85, May 2014.

[6]S. Yim and M. Sitti, "Design and Rolling Locomotion of a Magnetically Actuated Soft Capsule Endoscope," IEEE Transactions on Robotics, vol. 28, pp. 183-194, Feb. 2012.

[7]S. Yim, E. Gultepe, D. Gracias, and M. Sitti, "Biopsy using a Magnetic Capsule Endoscope Carrying, Releasing and Retrieving Untethered Microgrippers," IEEE Trans. on Biomedical Engineering, Sep. 2013.

[8]J. L. Gorlewicz, S. Battaglia, B. F. Smith, G. Ciuti, J. Gerding, A. Menciassi, K. L. Obstein, P. Valdastri, and R. J. Webster, "Wireless Insufflation of the Gastrointestinal Tract," IEEE Trans. Biomedical Engineering, vol. 60, May. 2013.

[9]R. Goffredo, D. Accoto, and E. Guglielmelli, "Swallowable smart pills for local drug delivery: present status and future perspectives," Expert Review of Medical Devices, vol. 12, pp. 585-599, Jun 2015.

[10] G. S. Lien, C. W. Liu, J. A. Jiang, C. L. Chuang, and M. T. Teng, "Magnetic Control System Targeted for Capsule Endoscopic Operations in the Stomach-Design, Fabrication, and in vitro and ex vivo Evaluations," IEEE Transactions on Biomedical Engineering, vol. 59, pp. 2068-2079, Jul. 2012.

[11] Z.-J. Sun, X.-G. Cheng, S. Cao, B. Ye, H.-H. Zhang, and S. Liu, "Multi-applications of a Magnet Configuration in Actuating Capsule Endoscope," IEEE/ASME International Conference on Advanced Intelligent Mechatronics (AIM), pp. 106-111, July 8-11 2014.

[12] Z.-J. Sun, B. Ye, Y. Qiu, X.-G. Cheng, H.-H. Zhang, and S. Liu, "Preliminary Study of a Legged Capsule Robot Actuated Wirelessly by Magnetic Torque," IEEE Transactions on Magnetics, vol. 50, August 2014. [13] A. W. Mahoney and J. J. Abbott, "Generating Rotating Magnetic Fields With a Single Permanent Magnet for Propulsion of Untethered Magnetic Devices in a Lumen," IEEE Transactions on Robotics, vol. 30, pp. 411-420, Apr. 2014.

[14] A. W. Mahoney and J. J. Abbott, "Five-degree-of-freedom manipulation of an untethered magnetic device in fluid using a single permanent magnet with application in stomach capsule endoscopy," The International Journal of Robotics Research, vol. 35, pp. 129-147, Jan. 2016. [15] N. D. Nelson and J. J. Abbott, "Generating Two Independent Rotating Magnetic Fields with a Single Magnetic Dipole for the Propulsion of Untethered Magnetic Devices," IEEE International Conference on Robotics and Automation, pp. 4056-4061, May 2015.

[16] M. Simi, M. Silvestri, C. Cavallotti, M. Vatteroni, P. Valdastri, A. Menciassi, and P. Dario, "Magnetically Activated Stereoscopic Vision System for Laparoendoscopic Single-Site Surgery," IEEE/ASME Transactions on Mechatronics, vol. 18, pp. 1140-1151, Jun. 2013.

[17] F. Munoz, G. Alici, and W. Li, "A Magnetically Actuated Drug Delivery System for Robotic Endoscopic Capsules," ASME Journal of Medical Devices, vol. 10, Mar. 2016.

[18] D. C. Jiles, "Introduction to magnetism and magnetic materials," Second Edition, Jun. 1998.

[19] F. Munoz, G. Alici, W. Li, and M. Sitti, "Size Optimization of a Magnetic System for Drug Delivery with Capsule Robots," IEEE Transactions on Magnetics, pp. 1-12, Jan. 2016. 